\title{
Correction to: Regulation of SIRT1/AMPK axis is critically involved in gallotannin-induced senescence and impaired autophagy leading to cell death in hepatocellular carcinoma cells
}

\author{
Hee Young Kwon ${ }^{1}$ Ju-Ha Kim ${ }^{1} \cdot$ Bonglee Kim ${ }^{1}$. Sanjay K. Srivastava ${ }^{2} \cdot$ Sung-Hoon Kim ${ }^{1}$
}

Published online: 16 July 2018

๑) Springer-Verlag GmbH Germany, part of Springer Nature 2018

Correction to: Arch Toxicol (2018) 92:241-257

https://doi.org/10.1007/s00204-017-2021-y

This article contains an error in AMPK isomer labeling in Fig. 4. AMPK $\alpha 1$ labeled in Fig. 4 and main text should be corrected as AMPK $\alpha$, since this antibody (\#2535 from Cell Signaling) can detect both AMPK $\alpha 1$ and $\alpha 2$. We are sorry for any inconvenience caused.

The original article can be found online at https://doi.org/10.1007/ s00204-017-2021-y.

Sung-Hoon Kim

sungkim7@khu.ac.kr

1 Cancer Molecular Targeted Herbal Research Center, College of Korean Medicine, Kyung Hee University, 1 Hoegi-dong, Dongdaemun-gu, Seoul 130-701, Republic of Korea

2 Department of Biomedical Sciences and Cancer Biology Center, Texas Tech University Health Sciences Center, Amarillo, TX 79106, USA 